\title{
Travelling front of a decaying brane in string field theory
}

\author{
Debashis Ghoshal and Preeda Patcharamaneepakorn \\ School of Physical Sciences, Jawaharlal Nehru University, \\ New Delhi 110067, India \\ E-mail: dghoshal@mail.jnu.ac.in, preeda.pat@gmail.com
}

\begin{abstract}
We consider the inhomogeneous decay of an unstable D-brane of bosonic string theory in a linear dilaton background in a light-cone frame. At the lowest level, the dynamical equation that describes this process is a generalisation (that includes nonlocality and time delay) of a reaction-diffusion equation studied by Fisher (and others). We argue that the equation of motion of the cubic open string field theory is satisfied at least to the second order when we start with this 'Fisher deformation', a marginal operator which has a simple pole term in its OPE. We also compute the one-point functions of closed string operators on the disc in the presence of this deformation.
\end{abstract}

KeYwords: Tachyon Condensation, D-branes, String Field Theory

ARXIV EPRINT: 1307.4890 


\section{Contents}

1 Introduction 1

2 Travelling front to the tachyon equation: a review 2

3 Travelling front in OSFT 4

3.1 Solution at second order 5

$\begin{array}{ll}3.2 & \text { Gauge condition }\end{array}$

3.3 Comments on higher order correction 8

4 Disc partition function $\quad 8$

5 Summary and comments 12

\section{Introduction}

The study of tachyonic instabilities in configurations of D-branes in string theory is an important one [1]. While time-dependent processes in string theory are difficult to analyze in general, the process of tachyon rolling down the potential is one in which certain aspects of dynamics have been found to be tractable [2-7]. In particular, the pioneering studies in ref. [2] found solutions of the equations of motion of cubic, open string field theory that move away from the unstable vacuum, but also go past the stable vacuum and exhibit wild oscillations at late times. This is not unexpected in the absence of any coupling to the closed string modes, to which an unstable D-brane is expected to decay [1]. A complete treatment of this problem would require one to use interacting open-closed string field theory [8], the formulation of which is not well understood as yet (see, however, refs. [9, 10]).

An alternative, which avoids this complexity, is to put the open strings in the presence of a linear dilaton background, which of course originates in the closed string sector. This was suggested by the authors of ref. [3], who use light-cone coordinates $x^{ \pm}$, and consider the dilation profile linear in $x^{-}$. The underlying conformal field theory (CFT) is well understood: the dilaton, being linear along a null direction, changes the (world-sheet) conformal dimension of the vertex operators, but does not alter the matter contribution to the central charge. Ref. [3] studied the homogeneous decay ${ }^{1}$ of the tachyon as a function of light-cone time $x^{+}$, solved the equation of motion for the tachyon (zero-level truncation) and extended this to the equations of motion of the full open string field theory.

The case of inhomogeneous decay in this framework was considered in ref. [11]. At zero level truncation, the equation of motion of the tachyon (as a function of $\tau$ and one

\footnotetext{
${ }^{1}$ In the terminology adopted in this paper, 'homogeneous' refers to processes dependent on light-cone time $\left(x^{+}\right)$only. We will also use $x^{+} \equiv \tau$ to simplify notation.
} 
other coordinate $y$ along the brane) was found to have a close resemblance to the ubiquitous reaction-diffusion equation pioneered in refs. [12] (as quoted in [19]) and [13, 14]. Specifically, the non-linear reaction term of the 'Fisher equation for the tachyon on a decaying brane', eq. (2.3), involves a time delay and spatial averaging with a Gaussian kernel, hence it is non-local ${ }^{2}$ as well. Like its ancestor, the Fisher equation for the tachyon has a travelling front solution that separates the brane from the (closed string) vacuum and moves with a constant speed retaining its shape. This solution was found using a singular perturbation analysis.

In this paper, we discuss the extension of this travelling front to the equations of motion of open string field theory (which takes the effect of the higher stringy modes into account). Specifically, we start with the deformation corresponding to the front solution in ref. [11] - this is a marginal perturbation of the D-brane CFT. In fact, there is a continuous family of marginal operators, however, we shall see that only one of these allows for a solution to the equations at second order. It is the same one for which the front propagates with the minimum speed. Thus, in both situations this operator plays a special role. It seems likely that it is exactly marginal. We call the corresponding perturbation the Fisher deformation.

In the following, we start with a brief review of some relevant results from the literature. In section 3, we extend the marginal Fisher deformation to the equations of motion of string field theory to the second order in perturbation parameter. We comment on the gauge condition and the complications in extending to higher order terms. section 4 deals with the one-point function of closed string vertex operators in the presence of Fisher deformation of the boundary CFT. We end with a summary and some comments.

\section{Travelling front to the tachyon equation: a review}

Let us, for definiteness, consider the CFT corresponding to an unstable D $p$-brane of the bosonic string theory. The string field $|\Psi\rangle$ is a vector in the Hilbert space of the matterghost (boundary) CFT, and may be expanded as

$$
|\Psi\rangle=\phi(X) c|0\rangle+\cdots=\int \frac{d^{p+1} k}{(2 \pi)^{p+1}} \phi(k) e^{i k \cdot X} c_{1}|0\rangle+\cdots,
$$

where $\phi$ is the tachyon and the dots stand for the higher stringy modes. The Chern-Simons type action of the cubic open string field theory

$$
S=\frac{1}{g^{2}}\left(\frac{1}{2}\left\langle\Psi\left|Q_{B}\right| \Psi\right\rangle+\frac{1}{3}\langle\Psi \mid \Psi \star \Psi\rangle\right),
$$

is defined in terms of the $\star$-product, the BRST operator $Q_{B}$ and the inner product of the matter-ghost CFT. Let us retain only the tachyon field $\phi$ (level truncation to zeroth order) and further restrict to spatially homogeneous decay, i.e., $\phi$ depends only on time $t$. This analysis was done in detail in ref. [2], where it was found that the equation of

\footnotetext{
${ }^{2}$ Non-locality in reaction-diffusion systems has been considered in subsequent literature, mainly in Mathematical Biology. However, the specific form that appears in the tachyon equation of motion is distinct to our knowledge.
} 


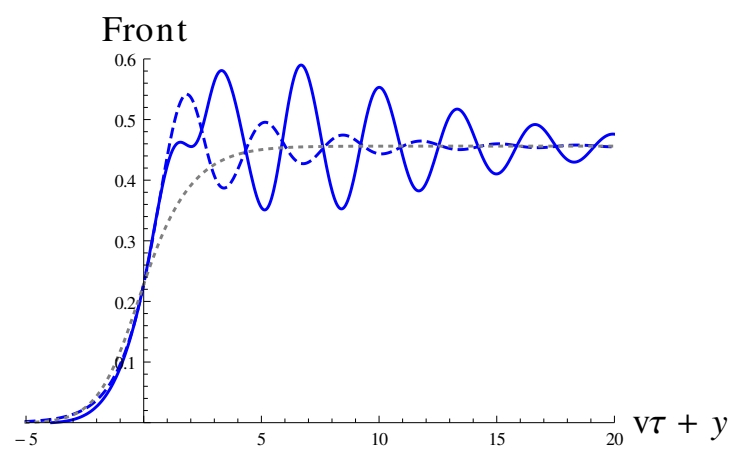

Figure 1. The interpolating solution $\phi(\tau)$ of the the homogeneous tachyon equation, which also solves the lowest order inhomogeneous equation, is the blue dashed curve. With the first corrections it is the solid blue curve. The solution of the ordinary Fisher equation (gray dotted) is shown for comparison. (The argument is only $\tau$ for the homogeneous case.)

motion has solutions that start at the maximum of the potential (at $\phi_{U}=0$ ) towards the (local) minimum (at $\phi=K^{-3} \simeq 0.456$ ), but overshoot and exhibit (non-linear) oscillations around the minimum. At late times, as a result of the non-local non-linear interactions, these behave wildly. However, these authors did not find a solution that interpolates between the D-brane and the (closed string) vacuum. Physically this is not unexpected, as the energy of the D-brane cannot dissipate into the closed string modes in a theory of open strings alone. This is, nevertheless, at odds with what is known from other approaches to D-brane decay pioneered by Sen (see also, refs. [15-18] in the context of string field theory).

Let us now consider a linear dilaton background $\mathcal{D}(x)=-D^{+} x^{-} \equiv-b x^{-}$(where $x^{ \pm}=(t \pm x) / \sqrt{2}$ and $b$ is a constant) following ref. [3]. The linear dilaton CFT is solvable. Moreover, since the dependence of the dilaton is along a null direction, the central charge of the matter CFT remains the same, Only the (world-sheet) conformal dimension of the tachyon vertex operators $e^{i k \cdot X}$ change from $k^{2}$ to $k^{2}+i b k^{-}$. Consequently, the equation of motion for the tachyon gets modified to (we have used $\alpha^{\prime}=1$ ):

$$
\left(b \frac{\partial}{\partial \tau}-\nabla_{\perp}^{2}\right) \phi\left(\tau, \mathbf{x}_{\perp}\right)=\phi\left(\tau, \mathbf{x}_{\perp}\right)-K^{3} e^{-2 \alpha b \partial_{\tau}+\alpha \nabla_{\perp}^{2}}\left[e^{\alpha \nabla_{\perp}^{2}} \phi\left(\tau, \mathbf{x}_{\perp}\right)\right]^{2},
$$

where $K=3 \sqrt{3} / 4, \alpha=\ln K$ and $\mathbf{x}_{\perp}$ denotes the coordinates along the D-brane that are transverse to the light-cone coordinates. This has been referred to as the 'Fisher equation for the tachyon on a decaying brane' in [11].

In homogeneous decay, $\phi=\phi(\tau)$ depends only on (light-cone) time, therefore, the equation simplifies [3, 5-7] to that of a delayed growth model [19]. The usual growth model (logistic equation) of population dynamics has a simple interpolating solution, but the delay leads to oscillations around the stable fixed point at $\phi_{S}$. The solution interpolating between these fixed points (see figure 1) was found in [3] (see also [11]).

Now consider the case of inhomogeneous decay governed by the Fisher equation (2.3). Let us, for simplicity, take the tachyon to depend only on one direction along the brane, and denote this by $y$ (it is transverse to the light-cone coordinates $x^{ \pm}$). There is a travelling front solution that moves, say, from right to left, so that at any instant of time the region 
to the right of the front moves towards the stable fixed point. If we linearize the equation around the maximum $\phi_{U}=0$, and put in the ansatz $\phi_{v} \sim \exp (k(y+v(k) \tau))$, we find the dispersion relation

$$
v(k)=\frac{1}{b}\left(k+\frac{1}{k}\right) .
$$

The wavenumber $k$ is real for $v(k) \geq v_{\min }=2 / b$, therefore, any of these solves the linearized equation. For a large class of nonlinear interactions, the travelling front of the usual Fisher-type equation (without delay or nonlocality), has been proven to select the front solution corresponding to $v_{\min }[19,20]$. Ref. [11] made the plausible assumption ${ }^{3}$ that this feature is also true of eq. (2.3). Then a travelling front solution to this equation in the form of $\phi(\tau, y)=\Phi(\eta=y+v \tau)$, was found by a straightforward adaptation of singular perturbation analysis $[19,20]$ in terms of the parameter $\varepsilon \equiv 1 / v^{2} b^{2} \leq 0.25$. At the leading order, i.e., $\mathcal{O}(1)$ in $\varepsilon$, the front is just as in figure 1 -higher order corrections, can be found systematically following ref. [11].

\section{Travelling front in OSFT}

The approach of ref. [11] outlined above, however, gives us a solution to the tachyon equation of motion (2.3), which is an approximate solution, being a level-zero truncation of the equation of motion of string field theory

$$
Q_{B}|\Psi\rangle+|\Psi \star \Psi\rangle=0 .
$$

We would now like to address the question of finding a solution to the above, starting with the tachyon vertex operator

$$
\phi_{k}=: \exp \left(k\left(Y+v(k) X^{+}\right)\right):
$$

with $v(k)$ as in eq. (2.4). Clearly, $\Psi=c \phi_{k}$ solves the linearized equation of motion $Q_{B}|\Psi\rangle=$ 0 . This is equivalent to the statement that $\phi_{k}$ is a marginal deformation of the underlying (boundary) CFT. Indeed, the dimension of $\phi_{k}$ (in the linear dilaton background) is

$$
h\left(\phi_{k}\right)=k_{\mu} k^{\mu}+i k_{\mu} D^{\mu}=-k^{2}+i(-i k v(k)) b=1
$$

for all values of $k$, thanks to the dispersion relation (2.4). (Let us note parenthetically that this is reminiscent of 'Liouville dressing' of matter vertex operators in non-critical string theory.) At this stage $\phi_{k}$ with any $k$ seems to be a good marginal deformation. However, we shall see later that the value $k=1$, for which $v(k=1)=v_{\min }$, and therefore, plays a special role in the travelling front solution to the Fisher equation of the tachyon, turns out to be special as a marginal deformation. We shall refer to $\phi_{k=1}$ as the Fisher deformation.

Let us note that the case of homogeneous decay is recovered with $k=0$ and $k v(k)=$ $1 / b$. The marginal deformation corresponding to the tachyon vertex operator $e^{X^{+} / b}$ in this

\footnotetext{
${ }^{3}$ The additional elements of delay and nonlocality do not affect the linearized analysis around the maximum of the tachyon potential. The 'leading edge' of the wave is determined by the 'mass' of the tachyon and the parameter $b$, as in the standard case.
} 
case is special in that its OPE with itself vanishes identically. This considerably simplifies the problem of extending this marginal deformation to a solution of the SFT equations of motion [3].

We shall closely follow the method (and the notations) of ref. [21] (see also [22-24] and e.g. $[25,26]$ for recent applications) in order to extend the marginal Fisher deformation (3.1) to a solution of the SFT equation of motion. To this end, let us define

$$
\Psi_{\lambda}=\sum_{n=1}^{\infty} \lambda^{n} \Psi^{(n)}=\lambda c \phi_{k}+\lambda^{2} \Psi^{(2)}+\cdots,
$$

which allows one to determine $\Psi^{(n)}$ iteratively from

$$
Q_{B} \Psi^{(n)}=-\sum_{m=1}^{n-1} \Psi^{(m)} \star \Psi^{(n-m)}
$$

and construct the solution as a power series in $\lambda$. However, this involves inverting $Q_{B}$. It is by now well established that the solution is best attempted in the sliver frame in the Schnabl gauge. Formally, it is given by

$$
\left\langle\omega, \Psi^{(n)}\right\rangle=\prod_{m=1}^{n-1} \int_{0}^{1} d t_{m}\left\langle f \circ \omega(0) c \phi_{k}(1) \mathcal{B} c \phi_{k}\left(1+t_{1}\right) \cdots \mathcal{B} c \phi_{k}\left(1+\sum_{m} t_{m}\right)\right\rangle
$$

evaluated on the surface $\mathcal{W}_{1+\sum t_{m}}$, a wedge state in the sliver frame. In the above, $\omega$ is a generic state in the Hilbert space and $f(z)=\frac{2}{\pi} \tan ^{-1}(z)$ is the conformal map from the upper half plane to the sliver.

This expression is formal because of possible singularities that can arise when two operator insertions collide. In the case of the homogeneous decay considered in [3], the null field $X^{+}$does not have a contraction with itself, as a result of which there is no singularity when the marginal operators collide, and the formal solution above is well defined. In the case of Fisher deformation however, the OPEs are singular and regularization is needed to make sense of (3.4).

\subsection{Solution at second order}

The first correction $\Psi^{(2)}$ obtained from (3.4) is ill-defined due to the singularity from $t \rightarrow 0$. Let us regularize this as

$$
\left\langle\omega, \Psi_{\text {reg }}^{(2)}\right\rangle=\lim _{\epsilon \rightarrow 0} \int_{2 \epsilon}^{1} d t\left\langle f \circ \omega(0) c \phi_{k}(1) \mathcal{B} c \phi_{k}(1+t)\right\rangle_{\mathcal{W}_{1+t}}
$$

The above has a finite part and a divergent part, so we write $\Psi_{\text {reg }}^{(2)}=\Psi_{\text {fin }}^{(2)}+\Psi_{\text {div }}^{(2)}$. The finite part is as in ref. [21], hence can be dealt with as in there. The divergent part comes from the region $\epsilon \rightarrow 0$, where we use the OPE of the vertex operators to write

$$
\begin{aligned}
\lim _{\epsilon \rightarrow 0} c \phi_{k}(1) \mathcal{B} c \phi_{k}(1+2 \epsilon) & = \\
& {\left[\frac{1}{(2 \epsilon)^{2 k^{2}}}: c \phi_{k}^{2}:+\frac{1}{(2 \epsilon)^{2 k^{2}-1}}\left(\frac{1}{2}: c \partial \phi_{k}^{2}:-: c \partial c \phi_{k}^{2} \mathcal{B}:+c \phi_{k}^{2} \mathcal{L}:\right)(1)+\cdots\right], }
\end{aligned}
$$


where $: \phi_{k}^{2}:=: e^{2 k\left(Y+v(k) X^{+}\right)}$: has dimension $2-2 k^{2}$. (Note that the argument of $v$ is $k$ and not $2 k$ - the momenta in the $x^{+}$direction add to $2 k v(k)$, hence using eqs. (3.2) and (2.4), $: \phi_{k}^{2}$ : is a relevant operator.) In passing, we also note a curiosity of these deformations: even though the perturbations are marginal, since $X^{+}$does not have a self-contraction, their behaviour in the OPE is like those of relevant ones - an exception being the Fisher deformation, which behaves like a truly marginal operator.

From the above, we find

$$
\Psi_{\mathrm{div}}^{(2)}(\epsilon)=\frac{c: \phi_{k}^{2}:}{\left(2 k^{2}-1\right)(2 \epsilon)^{2 k^{2}-1}}+\int_{2 \epsilon} \frac{d t}{t^{2 k^{2}-1}}\left(\frac{1}{2} c: \partial \phi_{k}^{2}:-c \partial c \mathcal{B}: \phi_{k}^{2}:+c: \phi_{k}^{2}: \mathcal{L}\right)+\cdots
$$

There is no divergence for momenta in the range $k^{2}<1 / 2$. For $k^{2}=1 / 2$, the first term is logarithmically divergent, therefore, the renormalization method will fail. Both the terms are divergent for $\frac{1}{2}<k^{2}<1$, while for $k^{2}=1$, namely for the Fisher deformation, there is a logarithmic divergence in the second term. Finally, for $k^{2}>1$, there will be additional divergent terms.

We shall return to the first term, but first we want to check if the second term is BRST-exact. Indeed it is easy to check that

$$
\begin{aligned}
\frac{1}{2} c: \partial \phi_{k}^{2}:-c \partial c \mathcal{B}: \phi_{k}^{2}:+c: \phi_{k}^{2}: \mathcal{L}=Q_{B} & \left(\frac{1}{2}: \phi_{k}^{2}:-c \mathcal{B}: \phi_{k}^{2}:\right) \\
& +\left(2 k^{2}-2\right)\left(\frac{Q_{B}\left(c: \phi_{k}^{2}:\right) \mathcal{B}}{2 k^{2}-1}+\frac{1}{2} \partial c: \phi_{k}^{2}:\right) .
\end{aligned}
$$

i.e., a part of it is independent of $k$ and BRST-exact, however, there is in general also a $k$-dependent piece which spoils BRST-exactness. The only exception is the case of Fisher deformation $\phi_{k=1}$, for which the second term vanishes, therefore, the operator is BRSTexact. At this point we can use an ambiguity in $\Psi^{(2)}$. As one can see from eq. (3.3) it is defined only upto a BRST-closed term. Therefore, we are free to add to it $\Psi_{\text {exact }}^{(2)}$, a BRST-exact term defined as

$$
\Psi_{\text {exact }}^{(2)}=-\int_{2 \epsilon} \frac{d t}{t^{2 k^{2}-1}} Q_{B}\left(\frac{1}{2}: \phi_{k}^{2}:-c \mathcal{B}: \phi_{k}^{2}:\right)
$$

to remove the second divergent term in eq. (3.7).

As for the first divergent term, following [21], we notice that the regularized expression (3.5) does not satisfy the SFT equation of motion due to the presence of the surface term

$$
\begin{aligned}
\langle f \circ \omega(0) & \left.c: \phi_{k}:(1) c: \phi_{k}:(1+2 \epsilon)\right\rangle_{W_{1+2 \epsilon}} \\
& =\left\langle f \circ \omega(0) Q_{B}\left(\frac{1}{(2 \epsilon)^{2 k^{2}-1}} \frac{c: \phi_{k}^{2}:(1+\epsilon)}{2 k^{2}-1}+\frac{\mathcal{B} c \partial c: \phi_{k}^{2}:(1+\epsilon)}{(2 \epsilon)^{2 k^{2}-2}}+\cdots\right)\right\rangle_{\mathcal{W}_{1+2 \epsilon}} \\
& \equiv-\left\langle f \circ \omega(0) Q_{B} \Psi_{\mathrm{CT}}^{(2)}(\epsilon)\right\rangle .
\end{aligned}
$$


Consequently,

$$
\Psi_{\mathrm{CT}}^{(2)}(\epsilon)=-\frac{1}{\left(2 k^{2}-1\right)(2 \epsilon)^{2 k^{2}-1}} c: \phi_{k}^{2}:(1+\epsilon)
$$

(defined upto terms which are regular for $\frac{1}{2}<k^{2} \leq 1$ ) may be used as a counter-term in defining $\Psi^{(2)}$. This counter-term exactly cancels the the divergent first term in eq. (3.7). Hence, the renormalized string field

$$
\Psi_{\text {ren }}^{(2)}=\lim _{\epsilon \rightarrow 0}\left(\Psi_{\text {reg }}^{(2)}+\Psi_{\text {exact }}^{(2)}+\Psi_{\mathrm{CT}}^{(2)}\right)
$$

is finite and satisfies the equation of motion to $\mathcal{O}\left(\lambda^{2}\right)$.

Although we are able to follow the steps in ref. [21] closely, the situation here is different from the one considered there. The general formalism assumes that the OPE of the marginal deformation is either regular, or has a double pole, but not a simple pole term. In contrast, the OPE of the Fisher deformation does have a simple pole term. Thankfully, however, the corresponding operator turns out to be BRST-exact (see eq. (3.8) above and the remarks in [21]), hence the ideas developed there also work in this case.

\subsection{Gauge condition}

The solutions outlined in ref. [21] break the (Schnabl) gauge condition. This is also the case for the solution seeded by the Fisher deformation. Specifically, the counter-term $\Psi_{\mathrm{CT}}^{(2)}$ breaks the gauge condition.

First, note that $\Psi_{\text {reg }}^{(2)}$ and $\Psi_{\text {exact }}^{(2)}$ satisfy the gauge condition: $B \Psi_{\text {reg }}^{(2)}=0$ and $B \Psi_{\text {exact }}^{(2)}=$ 0 , where $B$ is the zero mode of the anti-ghost in the sliver frame. Recall that $c=\frac{2}{\pi} c_{1}$, and $B^{+} \equiv B+B^{b}$, where $B^{b}$ is BPZ conjugate of $B$. Using the short-hand $: \phi_{k=1}^{2}: \equiv \phi_{F}^{2}$, the counter-term $\Psi_{\mathrm{CT}}^{(2)}$, can be written as

$$
\Psi_{\mathrm{CT}}^{(2)}=-\frac{1}{\pi \epsilon} e^{\epsilon L^{+}} c_{1} \phi_{F}^{2}|0\rangle=-\frac{1}{\pi \epsilon} c_{1} \phi_{F}^{2}|0\rangle+\frac{1}{\pi} L^{+} c_{1} \phi_{F}^{2}|0\rangle+\mathcal{O}(\epsilon),
$$

where $L$ and $L^{b}$ are the zero mode of the energy-momentum tensor and its BPZ conjugate in the sliver frame, and $L^{+} \equiv L+L^{b}$. It is not difficult to check that $L^{+} \phi_{F}^{2}=0$. This is due to fact that the conformal dimension of $\phi_{F}^{2}$ is zero. Applying $B$ to the counter-term

$$
B \Psi_{\mathrm{CT}}^{(2)}=\frac{1}{\pi} B^{+} c_{1} \phi_{F}^{2}|0\rangle \neq 0,
$$

we see that the gauge condition is violated by the counter-term. One can again take advantage of the ambiguity in the solution of (3.3) and add a BRST-closed state $\Xi$ and try to restore the gauge condition. If such a state exists, then $B\left(L^{+} c_{1} \phi_{F}^{2}|0\rangle+\Xi\right)=0$. When applied with $Q_{B}$, we find

$$
-L \Xi=L L^{+} c_{1} \phi_{F}^{2}|0\rangle-B Q_{B} L^{+} c_{1} \phi_{F}^{2}|0\rangle=-B Q_{B} L^{+} c_{1} \phi_{F}^{2}|0\rangle,
$$

where in addition to the standard relations $\left\{Q_{B}, B\right\}=L$ and $\left[L, L^{+}\right]=L^{+}$, we have used $L c_{1} \phi_{F}^{2}|0\rangle=-c_{1} \phi_{F}^{2}|0\rangle$. Once again, the right hand side is in the kernel of $L$, since $L L^{+} c_{1} \phi_{F}^{2}|0\rangle=0$. In fact, the only difference from ref. [21] is in the appearance of $\phi_{F}^{2}$ in 
place of the identity operator. However, this does not change anything because it is also a dimension-zero operator.

The formal solution $\Psi^{(n)}=\frac{B}{L} \Phi^{(n)}$ in the Schnabl gauge is well-defined when $\Phi^{(n)}$ does not have an overlap with the kernel of $L$. Moreover, $\Phi^{(2)}$ must be even under ghost-twist because $\Psi^{(1)}$ is even. There is a ghost-number two, even, BRST-exact term of dimension zero

$$
L^{+} c_{1} c_{0} \phi_{F}^{2}|0\rangle=Q_{B} L^{+} c_{1} \phi_{F}^{2}|0\rangle
$$

in the subspace of states for $\Phi^{(2)}$. This term is not annihilated by $B$, therefore, breaks the gauge condition.

\subsection{Comments on higher order correction}

The computations for $\Psi^{(3)}$ at the next order (and beyond) get rapidly very complicated, even for the Fisher deformation. This is due to the non-vanishing simple pole term in its OPE. As a result, the most singular term in the OPE of $\phi_{F}$ and $\phi_{F}^{2}$ has a fourth order pole! More generally,

$$
: \phi_{k}^{2}:(z): \phi_{k}:(w)=\frac{1}{(z-w)^{4 k^{2}}}: \phi_{k}^{3}:(w)+\frac{2}{3(z-w)^{4 k^{2}-1}} \partial_{w}: \phi_{k}^{3}:(w)+\cdots
$$

Consequently, while renormalizing the formal solution, we encounter

$$
: c \phi_{k}^{2}:(1+\epsilon) c \phi_{k}:(1+4 \epsilon)=\frac{1}{(3 \epsilon)^{4 k^{2}-1}}: c \partial c \phi_{k}^{3}:+\cdots
$$

The corresponding operator for the Fisher deformation is, however, BRST-exact: $c \partial c \phi_{F}^{3}=$ $\frac{1}{4} Q_{B}\left(c \phi_{F}^{3}\right)$. A counting of dimensions show that the dimensions of the operators accompanying all the singular terms are non-zero (negative) integers. In particular, the OPEs of $\phi_{F}$ and $\phi_{F}^{2}$ do not produce another marginal operator. This is also the case for the OPEs of $\phi_{F}^{n} \sim: e^{n k\left(Y+v(k) X^{+}\right)}$: (the dimension of which is $-n(n-2)$ ) at higher order, from the same dimension counting argument. Thus, it seems possible that all the singular terms are BRST-exact for the Fisher deformation, hence a renormalized solution may be found. However, we shall not attempt to prove this here.

\section{Disc partition function}

We shall now turn to the computation of the partition function on the disc with an insertion of a closed string vertex operator in the interior, in the presence of the Fisher deformation of the boundary CFT. This computation has been done many times in the past, e.g., in refs. $[27,28]$ for timelike tachyon (with or without additional spatial dependence) and in refs. $[3,7]$ for lightlike tachyon in a linear dilaton background. One can make use of the result in different ways: the partition function on the disc is closely related to the (space-time) action in the boundary string field theory approach $[29,30]$ - the two actually coincide on-shell. Moreover, one can get the energy-momentum tensor [31] by choosing the graviton vertex operator in the interior. 
Let us first calculate the one-point function of a closed string tachyon vertex operator with the momentum $p_{\mu}=\left(p_{+}, p_{-}, 0\right)$

$$
V_{\text {closed }}(X) \equiv V\left(p_{+}, p_{-}\right)=e^{p_{+} X^{+}+p_{-} X^{-}}
$$

on the disc $\left\langle V\left(p_{+}, p_{-}\right)\right\rangle_{\text {Disc }}$ in the background of the lightlike linear dilaton, in the presence of the perturbation $\int \phi_{k}(X)$ to the boundary CFT. We separate the zero modes $x^{\mu}$, and fix these with the using $\delta\left(x^{\mu}-\int \frac{d \theta}{2 \pi} X^{\mu}\right)$. Essentially we do the computation in [3, 7] for the deformation $\phi_{k}=: e^{k\left(Y+v(k) X^{+}\right)}$:, and also use the normal ordering described there. The result, from conformal perturbation theory, is

$$
\begin{aligned}
& \left\langle V\left(p_{+}, p_{-}\right)\right\rangle_{D} \\
& \quad=\sum_{n=0}^{\infty} \frac{(-\lambda)^{n}}{n !}\left(\prod_{i=1}^{n} \int_{0}^{2 \pi} \frac{d \theta_{i}}{2 \pi}\right)\left\langle V\left(p_{+}, p_{-}\right) \prod_{i=1}^{n}: e^{k\left(Y+v(k) X^{+}\right)}:\right\rangle \\
& \quad=e^{p_{+} x^{+}+p_{-} x^{-}} \sum_{n=0}^{\infty} \frac{(-\lambda)^{n}}{n !} e^{n k\left(v(k) x^{+}+y\right)}\left(\prod_{i=1}^{n} \int_{0}^{2 \pi} \frac{d \theta_{i}}{2 \pi}\right) \prod_{1 \leq i<j \leq n}\left|e^{i \theta_{i}}-e^{i \theta_{j}}\right|^{-2 k^{2}} .
\end{aligned}
$$

Notice that the power of the separation between the points of the boundary of the disc is negative. (As commented below eq. (3.6), the behaviour of these marginal perturbations sometimes resemble that of relevant operators, due to the presence $X^{+}$, which has trivial Wick contraction. Moreover, the contraction between the $X^{-}$part in the bulk and the $X^{+}$'s on the boundary are in powers of $\left|e^{i \theta}\right|$.) As a result, the integrand diverges whenever two (or more) boundary operators coincide. This is unlike in refs. [3, 7] and ref. [27]. In the case of the former, the integrand is trivial, because the lightlike tachyon deformation does not have a non-zero self-contraction, while, in the latter case of timelike tachyon, the power is positive.

The divergence in expression (4.1) needs to be regulated. One way would be to use point splitting, which would break conformal invariance. This requires one to proceed carefully. Instead, we recall that integrals of this type appear in the theory of random matrices (RMT). Indeed the authors of ref. [27, 36-38] used this observation to evaluate their integrals. In the context of RMT, these are known as Dyson's integrals, the values of which were conjectured by Dyson [32] to be

$$
\mathcal{D}_{n}(\beta)=\left(\prod_{i=1}^{n} \int_{0}^{2 \pi} \frac{d \theta_{i}}{2 \pi}\right) \prod_{1 \leq i<j \leq n}\left|e^{i \theta_{i}}-e^{i \theta_{j}}\right|^{2 \beta}=\frac{\Gamma(1+n \beta)}{(\Gamma(1+\beta))^{n}}
$$

and were proved in refs. [33-35]. The function $\mathcal{D}_{n}(\beta)$ is a meromorphic function of $\beta$. For the tachyon deformation considered here, we have $\mathcal{D}_{n}\left(-k^{2}\right)=\Gamma\left(1-n k^{2}\right) /\left(\Gamma\left(1-k^{2}\right)\right)^{n}$, leading to the series

$$
\sum_{n=0}^{\infty} \frac{(-\lambda)^{n}}{n !} \frac{\Gamma\left(1-n k^{2}\right)}{\left(\Gamma\left(1-k^{2}\right)\right)^{n}} e^{n k\left(y+v(k) x^{+}\right)} .
$$

For the homogeneous case, $k^{2}=0$, hence this is just the exponential series and known results [7] are recovered. For a general inhomogeneous decay, this is at best an asymptotic series: the numerator diverges at order $n=q$ for $k^{2}=p / q$. 

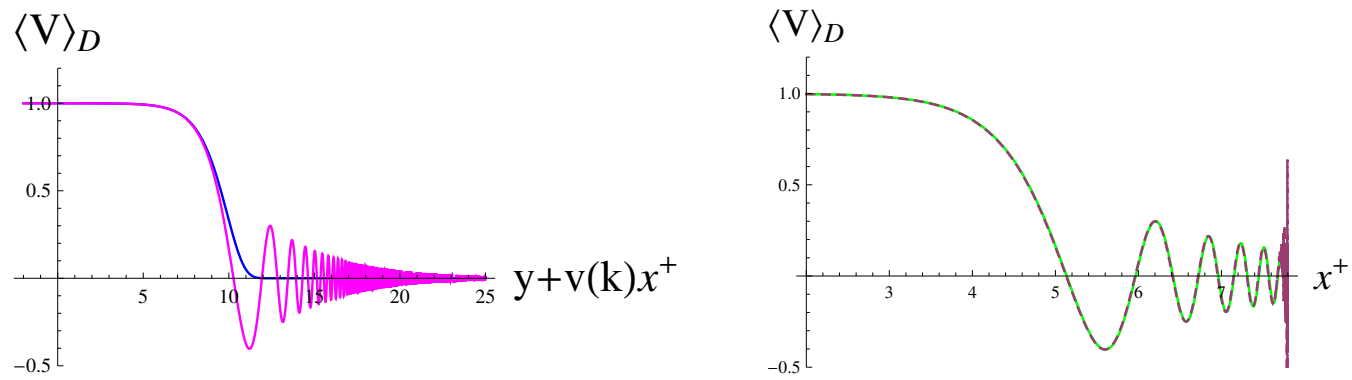

Figure 2. On the left: oscillatory decay of $\langle V\rangle_{D}$ as given by the Bessel function in eq. (4.5). The corresponding result for homogeneous decay is shown in solid blue. On the right: a comparison of the perturbative series with the expression (4.5). Notice that the series starts to behave badly for large values of the argument.

In the case of Fisher deformation $\left(k^{2}=1\right)$, both the numerator and the denominator are singular for $n \geq 2$, however, due to the presence of multiple factors of $\Gamma(0)$ in the denominator, the integrals actually vanish for $n \geq 2$ ! Taking this at face value, the only contributions are from the first two terms. This gives us $1-\lambda \exp \left(y+\frac{2}{b} x^{+}\right)$, which goes to 1 as the argument of the exponential goes to $-\infty$ (D-brane background) but diverges (to $-\infty)$ as it goes to $\infty$ (closed string vacuum). Clearly, this is not the expected behaviour.

Let us, instead, set $k^{2}=1+\varepsilon$, so that $\mathcal{D}_{n}\left(-k^{2}=-1-\varepsilon\right)=\frac{(\varepsilon)^{n-1}}{n !}$, using which we find

$$
\langle V\rangle_{D} \equiv\langle V(0,0)\rangle_{D} \sim \frac{1}{\varepsilon} \sum_{n=0}^{\infty} \frac{1}{(n !)^{2}}\left(-\varepsilon \lambda e^{y+\frac{2}{b} x^{+}}\right)^{n}=\frac{1}{\varepsilon} J_{0}\left(2 \sqrt{\varepsilon \lambda} e^{\left(y+\frac{2}{b} x^{+}\right) / 2}\right),
$$

where $J_{0}(z)$ is the Bessel function. We get a finite answer if we renormalize the coupling $\lambda_{R}=\varepsilon \lambda$. The additional factor of $\varepsilon$ may be absorbed in the coupling of the closed string vertex operator $V$. In terms of the renormalized couplings

$$
\langle\tilde{V}\rangle_{D} \sim J_{0}\left(2 \sqrt{\tilde{\lambda}} \exp \left\{\frac{1}{2}\left(y+\frac{2}{b} x^{+}\right)\right\}\right)
$$

which goes to one as the argument of the exponential goes to $-\infty$ (D-brane background) and settles to zero (after some oscillations - see figure 2) as it goes to $\infty$ (closed string vacuum).

Some comments are in order. First, we do not understand why the disc partition function oscillates around the closed string vacuum. As such these do not seem to be related to the oscillations in figure 1 - those are due to the time delay, and present also for the homogeneous decay - even though they are consistent with the oscillatory convergence of the tachyon. Secondly, while the renormalization gives a sensible result, it will be good to have a better understanding of its implications. Finally, if we approach $k^{2}=1$ from below, by parametrizing $k^{2}=1-\varepsilon$ instead, the Dyson's integrals alternate in sign: $\mathcal{D}_{n}\left(-k^{2}=-1+\varepsilon\right)=\frac{(-\varepsilon)^{n-1}}{n !}$. The additional sign cancels the alternating signs in the perturbation series in $\lambda$ in (4.3), and one ends up with the modified Bessel function of the first kind $I_{0}\left(2 \sqrt{\tilde{\lambda}} \exp \frac{1}{2}\left(y+\frac{2}{b} x^{+}\right)\right)$. This does not oscillate, but diverges as the argument 
becomes large. It is worth noting, however, that the integrands involved modulus-square of complex functions, thus are manifestly positive, a feature that the parametrization we have used, preserves.

Curiously disc partition function with oscillatory tachyon profile was considered earlier by Jokela et al.[36]. They considered a boundary perturbation by the OSFT solution of refs. [21, 22]. However, this solution is seeded by a timelike tachyon $e^{X^{0}}$. Consequently, singularities do not arise from colliding boundary operators - in other words $\beta$ (in eq. (4.2)) is positive. Nevertheless, these authors consider summing the perturbation series resulting from a complicated deformation that, in principle, has an infinite number of terms, and study its analytic properties. They concluded that although there are oscillations in the disc amplitude, these are suppressed at late times. This behavior is similar to what we find, even though the oscillations of the timelike tachyon (unlike that in the linear dilation background with lightlike or Fisher-type momentum) continue at late times.

Next we choose the zero-momentum graviton for the closed string vertex operator, and calculate the one-point function

$$
\mathcal{A}^{\mu \nu}=\left\langle: \partial X^{\mu} \bar{\partial} X^{\nu}:\right\rangle_{D}
$$

on the disc. This requires evaluation of the integrals

$$
\mathcal{A}_{n}(\beta)=\left(\prod_{i=1}^{n} \int_{0}^{2 \pi} \frac{d \theta_{i}}{2 \pi}\right) \prod_{1 \leq i<j \leq n}\left|e^{i \theta_{i}}-e^{i \theta_{j}}\right|^{2 \beta} \sum_{\ell, m} e^{-i\left(\theta_{\ell}-\theta_{m}\right)} .
$$

These integrals may be evaluated using the orthonormality of Jack polynomials [39, 40]. (When separated into diagonal and off-diagonal parts, the former reduces to the Dyson integral.) The result is

$$
\begin{aligned}
\mathcal{A}_{n}(\beta) & =\left(\prod_{i=1}^{n} \int_{0}^{2 \pi} \frac{d \theta_{i}}{2 \pi}\right) \prod_{1 \leq i<j \leq n}\left|e^{i \theta_{i}}-e^{i \theta_{j}}\right|^{2 \beta}\left(n+\sum_{\ell \neq m} e^{-i\left(\theta_{\ell}-\theta_{m}\right)}\right) \\
& =n \frac{\Gamma(1+n \beta)}{(\Gamma(1+\beta))^{n}}-\frac{\beta n(n-1)}{1+\beta(n-1)} \frac{\Gamma(1+n \beta)}{(\Gamma(1+\beta))^{n}} \\
& =\frac{n}{1+\beta(n-1)} \frac{\Gamma(1+n \beta)}{(\Gamma(1+\beta))^{n}}=\frac{n}{1+\beta(n-1)} \mathcal{D}_{n}(\beta) .
\end{aligned}
$$

This evidently agrees with the known result of ref. [27] for $\beta=1$. We shall consider the above to be analytic in $\beta$ and continue to negative ${ }^{4}$ values. In general, the associated series can be written as

$$
\mathcal{A}\left(-k^{2}\right)=\sum_{n} \frac{(-\lambda)^{n}}{n !} e^{n k\left(y+v(k) x^{+}\right)} \mathcal{A}_{n}\left(-k^{2}\right) .
$$

\footnotetext{
${ }^{4}$ The reciprocity relation for Gamma functions extends to the Beta functions and the Selberg integrals [41]. These are therefore well-defined for negative values of the parameters. Although we are not aware of such relations for the more general cases needed here, similar reciprocity relations are likely to be true.
} 

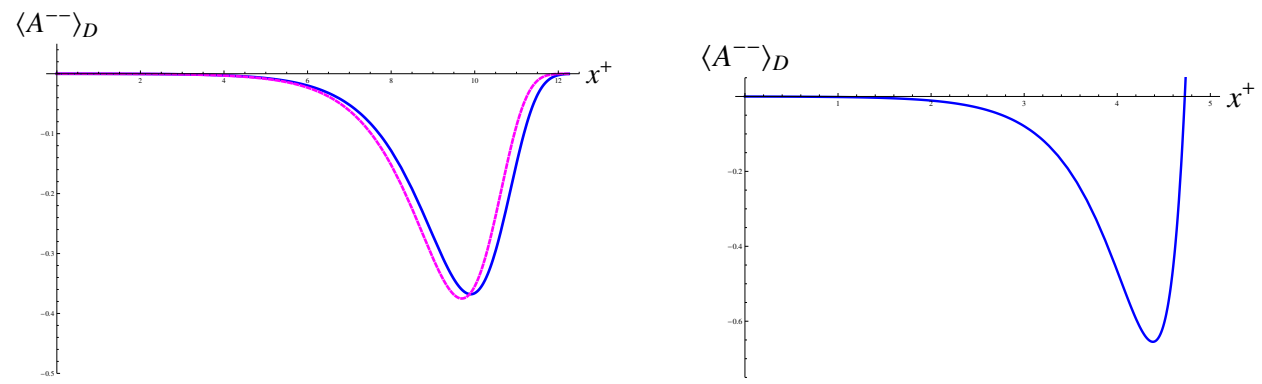

Figure 3. The function $\mathcal{A}^{--}\left(-k^{2}\right)$ as a consequence of eq. (4.8). On the left: the solid (blue) curve is for the exact expression of $\mathcal{A}^{--}(0)$ and the dashed (magenta) curve is for the series with a small value of $k^{2} \sim 0.02$. On the right: the plot of the series corresponding to the Fisher deformation, $k^{2}=1$. It diverges for large values of the argument.

In the case of Fisher deformation, $\beta=-k^{2}=-1$, therefore, the additional pre-factor in $\mathcal{A}_{2}$ is divergent. Writing $k^{2}=1+\varepsilon$ as before,

$$
\mathcal{A}_{2}=4 \frac{\Gamma(-1-2 \epsilon))}{(\Gamma(-\epsilon))^{2}}-1=2 \varepsilon-1
$$

We see that while the first term gives the expected form in terms of renormalized coupling $\lambda_{R}$ and renormalized closed string vertex operator, the second (constant) term will give a divergent contribution when written in terms of the renormalized quantities. This certainly is a cause of concern, and is perhaps due to the analytic continuation used. However, it is a problem for one term of the series and in the following, we shall omit this singular contribution and look at the rest of the series. Then, for the one-point function of the graviton vertex operators, we find that the non-vanishing components are $\mathcal{A}^{--} \sim k^{2} v^{2} \mathcal{A}\left(-k^{2}\right)$, $\mathcal{A}^{-y} \sim-k^{2} v \mathcal{A}\left(-k^{2}\right)$ and $\mathcal{A}^{y y} \sim k^{2} \mathcal{A}\left(-k^{2}\right)$. The behaviour of the function $\mathcal{A}^{--}$given by the series expansion is shown in figure 3. For large values of the argument, the series is seen to diverge.

Recall that the series for the tachyon one-point function converges to the Bessel function $J_{0}$. Motivated by this, we rewrite the expansion of $\mathcal{A}(-1)$ in terms of Bessel functions (using the Bessel expansion of $x^{n}$ ). This turns out to improve the behaviour of the series significantly. The graviton one-point function now shows oscillatory convergence (see figure 4) as in $\langle V\rangle_{D}$. We should add, however, that this is also an asymptotic series and diverges for very large values of the argument (not in the range of the graph).

We close by noting that the components of the energy-momentum tensor can be computed from these functions. However, in the presence of the linear dilation, a more careful analysis is needed as the Einstein metric differs from the string metric.

\section{Summary and comments}

In this paper, we have considered a class of marginal deformations corresponding to inhomogeneous decay of an unstable D-brane in the cubic open string field theory. They satisfy the linearized equation of motion, and one of these, which we call the Fisher deformation, gives a front solution that travels with a minimum speed. We consider, in detail, 


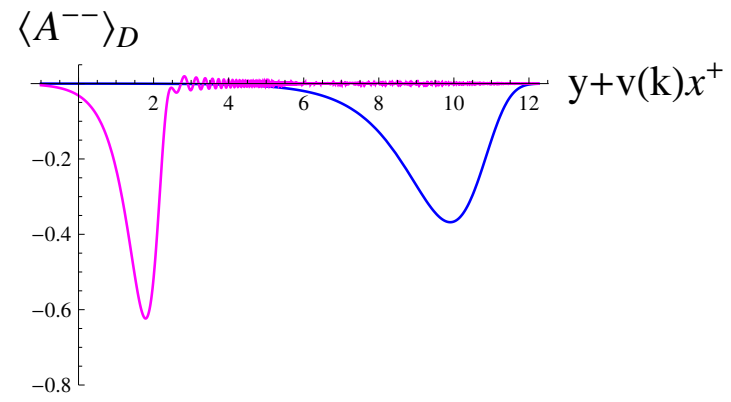

Figure 4. The function $\mathcal{A}^{--}\left(-k^{2}\right)$ when the series in eq. (4.8) is re-expressed in terms of Bessel functions. The solid magenta curve for $k^{2}=1$ shows oscillatory convergence. The solid blue curve for $k^{2}=0$ is also displayed for comparison.

the equations of OSFT to second order, and find that the Fisher deformation also solves the equations to this order. A characteristic of the marginal Fisher deformation is the appearance of a simple pole term in its OPE. Thankfully, however, the operator that accompanies this singular term turns out to be BRST-exact. This means that we are able to use the formalism developed for marginal operators. It is likely that this deformation is exactly marginal, however, the equations get rapidly very complicated and we leave the issue for future.

In the second part of our analysis, we computed the one-point functions of the closed string tachyon (in particular, the disc partition function) and the gravitons in the presence of the same marginal deformations on the boundary. These expressions involve UV singularities corresponding to coincident operators on the boundary. We have discussed one regularization scheme using Dyson and related integrals found in the context of random matrix theory. We propose a renormalisation that shows oscillations in the partition function before it decays to the closed string vacuum. This feature, the physical consequences of which may be worth exploring further, is in contrast to the case of homogeneous decay. There is also another spurious divergence at second order in the computation of the graviton one-point functions.

Finally, the regularization and renormalization provide an exact expression for the disc partition function in the background of the Fisher deformation. This may allow one to use the formalism of background independent open string field theory to get an alternative form of spacetime action for the tachyon field.

\section{Acknowledgments}

It is a pleasure to thank Camillo Imbimbo, Dushyant Kumar, Sunil Mukhi, Akhilesh Pandey, Ravi Prakash and Ashoke Sen. DG acknowledges the audience of a NORDITA seminar for many interesting questions and comments, and Fawad Hassan and Konstantin Zarembo for hospitality. We are grateful to Niko Jokela for useful correspondence and making us aware of some earlier work on the disc partition function. The work of DG was supported in part by SERC, DST (India) through the grant DST-SR/S2/HEP-043/2009, 
and PP is very grateful to Phra Jandee Jindatham and Watcharaporn Ladadok for inspiration and encouragement.

Open Access. This article is distributed under the terms of the Creative Commons Attribution License (CC-BY 4.0), which permits any use, distribution and reproduction in any medium, provided the original author(s) and source are credited.

\section{References}

[1] A. Sen, Tachyon dynamics in open string theory, Int. J. Mod. Phys. A 20 (2005) 5513 [hep-th/0410103] [INSPIRE].

[2] N. Moeller and B. Zwiebach, Dynamics with infinitely many time derivatives and rolling tachyons, JHEP 10 (2002) 034 [hep-th/0207107] [INSPIRE].

[3] S. Hellerman and M. Schnabl, Light-like tachyon condensation in Open String Field Theory, JHEP 04 (2013) 005 [arXiv: 0803.1184] [INSPIRE].

[4] L. Joukovskaya, Rolling solution for tachyon condensation in open string field theory, arXiv: 0803.3484 [INSPIRE].

[5] N. Barnaby, D.J. Mulryne, N.J. Nunes and P. Robinson, Dynamics and stability of light-like tachyon condensation, JHEP 03 (2009) 018 [arXiv:0811.0608] [INSPIRE].

[6] F. Beaujean and N. Moeller, Delays in open string field theory, arXiv:0912.1232 [INSPIRE].

[7] Y. Song, Note on light-like tachyon condensation, JHEP 07 (2010) 030 [arXiv:1003. 0230] [INSPIRE].

[8] B. Zwiebach, Oriented open-closed string theory revisited, Annals Phys. 267 (1998) 193 [hep-th/9705241] [INSPIRE].

[9] K. Ohmori, Toward open closed string theoretical description of rolling tachyon, Phys. Rev. D 69 (2004) 026008 [hep-th/0306096] [INSPIRE].

[10] A. Ishida and S. Teraguchi, On open-closed extension of boundary string field theory, JHEP 07 (2012) 059 [arXiv: 1204.6722] [INSPIRE].

[11] D. Ghoshal, Fisher equation for a decaying brane, JHEP 12 (2011) 015 [arXiv:1108.0094] [INSPIRE].

[12] R.-L. Luther, Raümliche Fortpflanzung Chemischer Reaktionen, Z. Elektrochem. Angew. P. 12 (1906) 506.

[13] R. Fisher, The wave of advance of advantageous genes, Ann. Eugenics 7 (1937) 355.

[14] A. Kolmogorov, I. Pertovsky and N. Piskunov, Etude de l'equation de la diffusion avec croissance de la quantité de matière et son application à un problème biologique, Bull. Moscow State Univ. Ser. A 1 (1937) 1.

[15] I. Ellwood, Singular gauge transformations in string field theory, JHEP 05 (2009) 037 [arXiv:0903.0390] [INSPIRE].

[16] L. Bonora, C. Maccaferri and D. Tolla, Relevant deformations in open string field theory: a simple solution for lumps, JHEP 11 (2011) 107 [arXiv:1009.4158] [INSPIRE].

[17] L. Bonora, S. Giaccari and D. Tolla, The energy of the analytic lump solution in SFT, JHEP 08 (2011) 158 [Erratum ibid. 04 (2012) 001] [arXiv:1105.5926] [INSPIRE]. 
[18] T. Erler and C. Maccaferri, Comments on lumps from RG flows, JHEP 11 (2011) 092 [arXiv: 1105.6057] [INSPIRE].

[19] J. Murray, Mathematical biology I: an introduction, $3^{\text {rd }}$ edition, Springer-Verlag, Berlin (2002).

[20] L. Debnath, Nonlinear partial differential equations for scientists and engineers, $2^{\text {nd }}$ edition, Birkhauser, Boston, U.S.A. (2004).

[21] M. Kiermaier, Y. Okawa, L. Rastelli and B. Zwiebach, Analytic solutions for marginal deformations in open string field theory, JHEP 01 (2008) 028 [hep-th/0701249] [INSPIRE].

[22] M. Schnabl, Analytic solution for tachyon condensation in open string field theory, Adv. Theor. Math. Phys. 10 (2006) 433 [hep-th/0511286] [INSPIRE].

[23] Y. Okawa, Comments on Schnabl's analytic solution for tachyon condensation in Witten's open string field theory, JHEP 04 (2006) 055 [hep-th/0603159] [INSPIRE].

[24] Y. Okawa, L. Rastelli and B. Zwiebach, Analytic solutions for tachyon condensation with general projectors, hep-th/0611110 [INSPIRE].

[25] B.-H. Lee, C. Park and D. Tolla, Marginal deformations as lower dimensional D-brane solutions in open string field theory, arXiv:0710.1342 [INSPIRE].

[26] O.-K. Kwon, Marginally deformed rolling tachyon around the tachyon vacuum in open string field theory, Nucl. Phys. B 804 (2008) 1 [arXiv:0801.0573] [InSPIRE].

[27] F. Larsen, A. Naqvi and S. Terashima, Rolling tachyons and decaying branes, JHEP 02 (2003) 039 [hep-th/0212248] [INSPIRE].

[28] N.D. Lambert, H. Liu and J.M. Maldacena, Closed strings from decaying D-branes, JHEP 03 (2007) 014 [hep-th/0303139] [INSPIRE].

[29] E. Witten, On background independent open string field theory, Phys. Rev. D 46 (1992) 5467 [hep-th/9208027] [INSPIRE].

[30] E. Witten, Some computations in background independent off-shell string theory, Phys. Rev. D 47 (1993) 3405 [hep-th/9210065] [INSPIRE].

[31] A. Sen, Rolling tachyon, JHEP 04 (2002) 048 [hep-th/0203211] [INSPIRE].

[32] F. Dyson, Statistical theory of the energy levels of complex systems. I, J. Math. Phys. 3 (1962) 140 [INSPIRE].

[33] J. Gunson, Proof of a conjecture by Dyson in the statistical theory of energy levels, J. Math. Phys. 3 (1962) 752.

[34] K. Wilson, Proof of a conjecture by Dyson, J. Math. Phys. 3 (1962) 1040 [inSPIRE].

[35] I. Good, Short proof of a conjecture by Dyson, J. Math. Phys. 11 (1970) 1884 [InSPIRE].

[36] N. Jokela, M. Jarvinen, E. Keski-Vakkuri and J. Majumder, Disk partition function and oscillatory rolling tachyons, J. Phys. A 41 (2008) 015402 [arXiv:0705.1916] [INSPIRE].

[37] N. Jokela, M. Jarvinen and E. Keski-Vakkuri, $N$-point functions in rolling tachyon background, Phys. Rev. D 79 (2009) 086013 [arXiv:0806.1491] [INSPIRE].

[38] N. Jokela, M. Jarvinen and E. Keski-Vakkuri, High-energy asymptotics of D-brane decay amplitudes from Coulomb gas electrostatics, Phys. Rev. D 82 (2010) 126009 [arXiv: 1008.4743] [INSPIRE]. 
[39] T. Baker and P. Forrester, Generalization of Morris integral, Duke Math. J. 95 (1998) 1.

[40] P.J. Forrester and S.O. Warnaar, The importance of the Selberg integral, Bull. Amer. Math. Soc. 45 (2008) 489 [arXiv:0710.3981].

[41] K. Mimachi and M. Yoshida, The reciprocity relation of the Selberg function, J. Comp. Appl. Math. 160 (2003) 209. 